\title{
EchoGéo
}

$8 \mid 2009$

Moyen-Orient : conflits et mobilités dans un espace mondialisé

\section{Boom aurifère et dynamiques économiques entre Sénégal, Mali et Guinée}

Faty B. Mbodj

\section{(2) OpenEdition}

\section{Journals}

Édition électronique

URL : https://journals.openedition.org/echogeo/11034

DOI : 10.4000/echogeo. 11034

ISSN : 1963-1197

Éditeur

Pôle de recherche pour l'organisation et la diffusion de l'information géographique (CNRS UMR 8586)

Référence électronique

Faty B. Mbodj, « Boom aurifère et dynamiques économiques entre Sénégal, Mali et Guinée », EchoGéo

[En ligne], 8 | 2009, mis en ligne le 25 mars 2009, consulté le 31 juillet 2021. URL : http://

journals.openedition.org/echogeo/11034; DOI : https://doi.org/10.4000/echogeo.11034

Ce document a été généré automatiquement le 31 juillet 2021.

EchoGéo est mis à disposition selon les termes de la licence Creative Commons Attribution - Pas d'Utilisation Commerciale - Pas de Modification 4.0 International (CC BY-NC-ND) 


\section{Boom aurifère et dynamiques économiques entre Sénégal, Mali et Guinée}

Faty B. Mbodj

\section{Introduction}

1 Après la Guinée (1995), incités par les institutions de Bretton Woods et la hausse des cours des ressources minières qui marque le contexte mondial de la fin des années 1990, certains pays comme le Mali (1999) et le Sénégal (2003) se dotent de nouveaux codes miniers. Ceux-ci ont créé un climat favorable à l'investissement de capitaux étrangers, via des compagnies minières d'origine canadienne, australienne et sudafricaine. En une dizaine d'années, plusieurs sociétés se sont installées dans ces pays pour exploiter les importantes mines d'or de Sabodala (à l'est du Sénégal), Loulo, Yatéla, Sadiola, Tabakoto (à l'ouest du Mali), Siguiri et Léro (au nord-est de la Guinée). Aux côtés des entreprises déjà en production, plusieurs dizaines de nouvelles venues s'investissent alors dans l'exploration de la zone. 


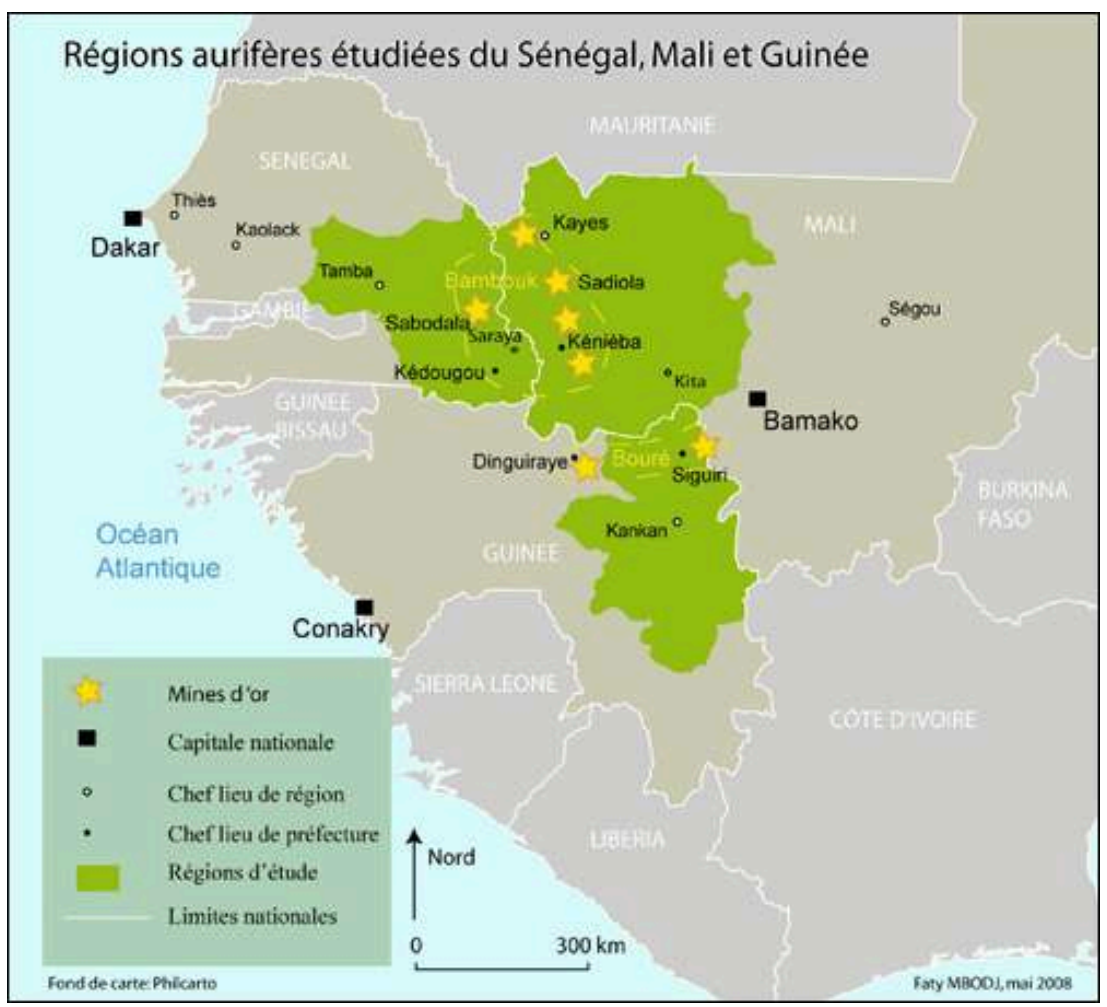

2 La présence des multinationales s'accompagne d'enjeux cruciaux à différentes échelles parce que leurs activités se déroulent dans des espaces transfrontaliers, correspondant aux anciennes provinces aurifères du Bouré et du Bambouk ${ }^{1}$, caractérisés depuis l'époque coloniale par l'enclavement, la pauvreté et l'exclusion. En effet, ces régions sont très éloignées de leurs capitales nationales respectives. Siguiri est à $794 \mathrm{~km}$ de Conakry; Kédougou à $720 \mathrm{~km}$ de Dakar tandis que Kayes et Bamako sont séparées par $598 \mathrm{~km}$. Il s'agit de zones difficilement accessibles en raison de l'éloignement et de la mauvaise qualité des routes, notamment pour la région de Tambacounda et celle de Kayes. La pauvreté est une caractéristique que partagent les territoires du Bouré et du Bambouk, souvent exclus des programmes de développement et d'aménagement, essentiellement concentrés sur les capitales et d'autres des espaces nationaux.

Pour ces différentes raisons, les espoirs portés par l'exploitation de type industriel sont considérables. Mais les transformations qu'elle pourrait induire sont-elles réellement susceptibles de concrétiser de tels espoirs, à l'échelle locale, à travers des dynamiques de développement, et à l'échelle sous régionale et nationale, par une meilleure intégration de ces espaces?

Nous appliquerons ce questionnement aux collectivités locales suivantes (voir carte) : la commune de Sadiola (région de Kayes, Mali), la communauté rurale de Khossanto (région de Tambacounda, Sénégal) et la communauté rurale de développement de Kintinian (communément appelée « Bouré $»-$ région de Kankan, Guinée Conakry). Ces trois collectivités se situent à des niveaux différents dans le processus d'extraction des ressources aurifères. Car si celles de Sadiola et de Siguiri ont débuté depuis plus d'une dizaine d'années (respectivement en 1997 et 1998), au Sénégal, l'expérience est beaucoup plus récente. La construction de la société d'exploitation australienne, MDL (Mineral Deposit Limited), a commencé en 2003 et l'exploitation en 2005. 
Carte 2 - Situation des collectivités locales étudiées

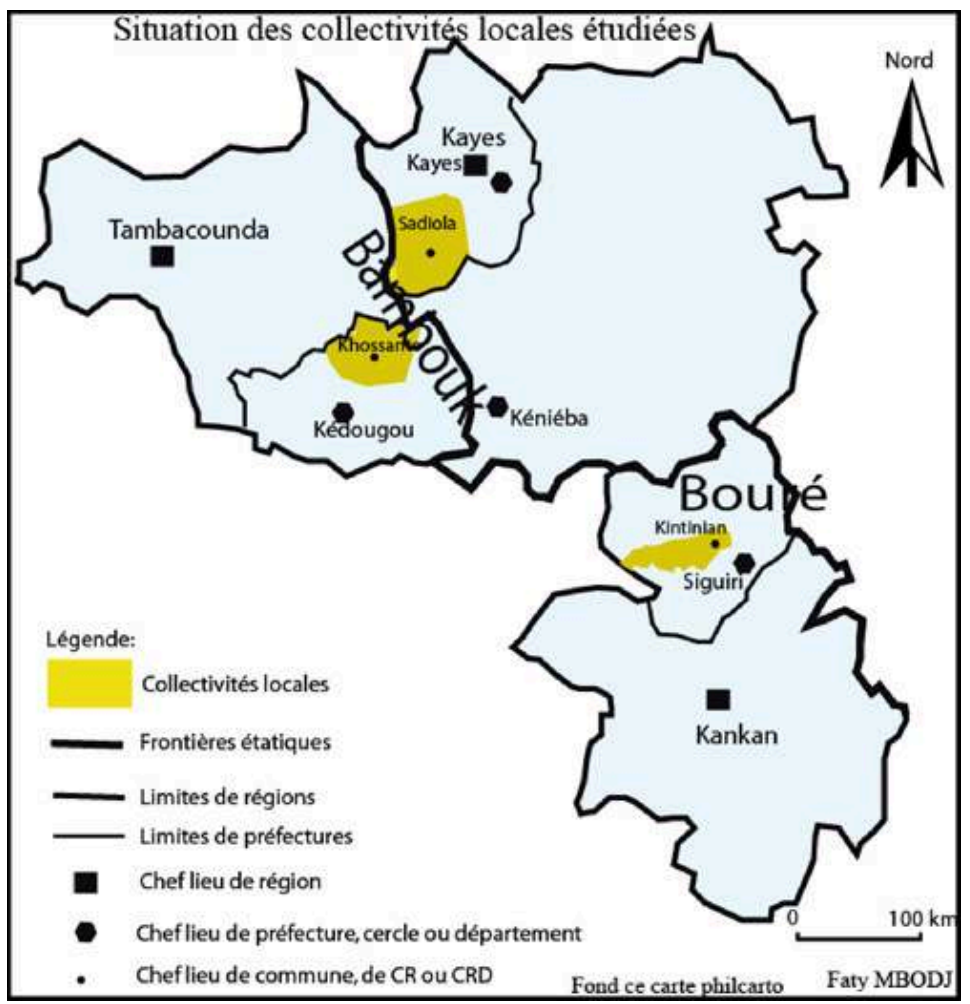

5 Dans la première partie de cet article nous présenterons le contexte économique des espaces choisis. Il permet de mettre en évidence l'importance des transformations directes et indirectes liées à l'implantation des multinationales, qui seront traitées dans la deuxième partie. La troisième partie analysera les interactions entre activités minières et autres types d'économies rurales telles que l'agriculture.

\section{Contextualisation économique des espaces étudiés}

Les régions étudiées ont en commun une histoire économique mouvementée. Après avoir été pendant plusieurs siècles des régions très dynamiques, elles ont été reléguées en marges du développement. Mais cette situation semble désormais évoluer vers une intégration économique et territoriale accrue grâce à un ensemble de facteurs, dont des dynamiques de restructuration des flux de personnes et de biens observées à l'échelle sous-régionale.

\section{Trajectoires économiques : entre dynamisme et marginalité}

7 Pour s'être positionnées au carrefour des échanges entre le Maghreb et l'Afrique noire, les régions aurifères du Bouré et du Bambouk constituaient l'un des grands pôles structurants du commerce transsaharien qui a atteint son âge d'or entre le VIII ${ }^{\mathrm{e}}$ et le $\mathrm{XV}^{\mathrm{e}}$ siècle. Elles étaient le lieu de négoce de divers produits venant du nord (chevaux, sel, perles, poteries, blé et orge, tissus, dattes, etc.) et du sud (gomme, esclaves, cola, ivoire, cuivre, peaux d'animaux, mil, œufs et plumes d'autruche, etc.). L'élément le plus important de ce commerce historique était l'or, issu des gisements qu'elles abritaient. 
Car, au-delà de sa valeur marchande, ce minerai était au cœur des processus de construction et de destruction des grandes institutions étatiques précoloniales (les empires du Mali, du Ghana, Songhaï etc.), des conquêtes territoriales que celles-ci menaient, ainsi que de leur rapport au reste du monde, notamment envers les Etats arabes de l'époque (Girard, 1992). Ces régions étaient ainsi politiquement convoitées et économiquement dynamiques.

Toutefois, ce statut privilégié fut suivi d'une phase de retournement de territoire (Ninot, 2003) qui les a placées dans une position de marginalité démographique, économique et géographique. Pour ne citer qu'un exemple, la région de Tambacoundaa la deuxième plus faible densité routière du pays (Ninot, op.cit). La route par laquelle on y accède n'a été goudronnée qu'en 1980 et les routes existantes sont mal entretenues car éloignées des centres de décision (Dakar). L'électricité aussi fait défaut: jusqu'en juillet 2000, Tambacounda n'était toujours pas raccordée au réseau national, seulement alimentée par deux groupes électrogènes de capacité insuffisante. La situation sanitaire de la région est également très mauvaise. La mortalité infanto-juvénile y est estimée à 182 pour mille contre 145,3 pour l'ensemble du Sénégal. En 1996 la région comptait en tout 195 postes de santé de proximité (cases et postes de santé), soit un poste pour $305 \mathrm{~km}^{2}$ contre 1 pour $91 \mathrm{~km}^{2}$ en moyenne dans le pays (PNUD, 2001).

La situation de pauvreté qui caractérise ces régions est corrélée au mode d'organisation territoriale de l'administration coloniale, surtout orienté vers les zones côtières. L'élite africaine postcoloniale a, elle aussi, pendant longtemps poursuivi cette même logique économico-territoriale (Mbodj, 1992 ; Ninot, 2003). Les conditions économiques de ces marges n'en sont que davantage dégradées. En outre, essentiellement structurées autour des cultures de rente, elles souffrent depuis les années 1970, et notamment 1990, des perturbations que celles-ci subissent, en raison des conditions climatiques et des fluctuations des cours mondiaux des matières premières.

C'est donc dans cette conjoncture difficile que les activités extractives interviennent. Mais elles débutent par ailleurs dans un contexte d'émergence de facteurs de mutation qu'il convient de mentionner, parce qu'ils contribuent à amplifier les impacts de l'exploitation minière.

\section{Contexte sous régional de l'exploitation industrielle : l'émergence de facteurs d'évolution}

11 Les espaces en marge étudiés sont, depuis quelques années, l'objet d'importantes mutations favorables à leur intégration nationale et sous-régionale. Elles tiennent à des initiatives gouvernementales mais aussi au rôle d'organisations inter-étatiques (NEPAD, Nouveau partenariat pour le développement de l'Afrique, et UEMOA, Union économique et monétaire ouest-africaine) qui se concrétise par la construction de routes transnationales financées par l'Union européenne, la Banque africaine de développement et le Fonds Saoudien (PER, 2006). 
Carte 3 - Des routes transnationales facteurs d'intégration sous régionale

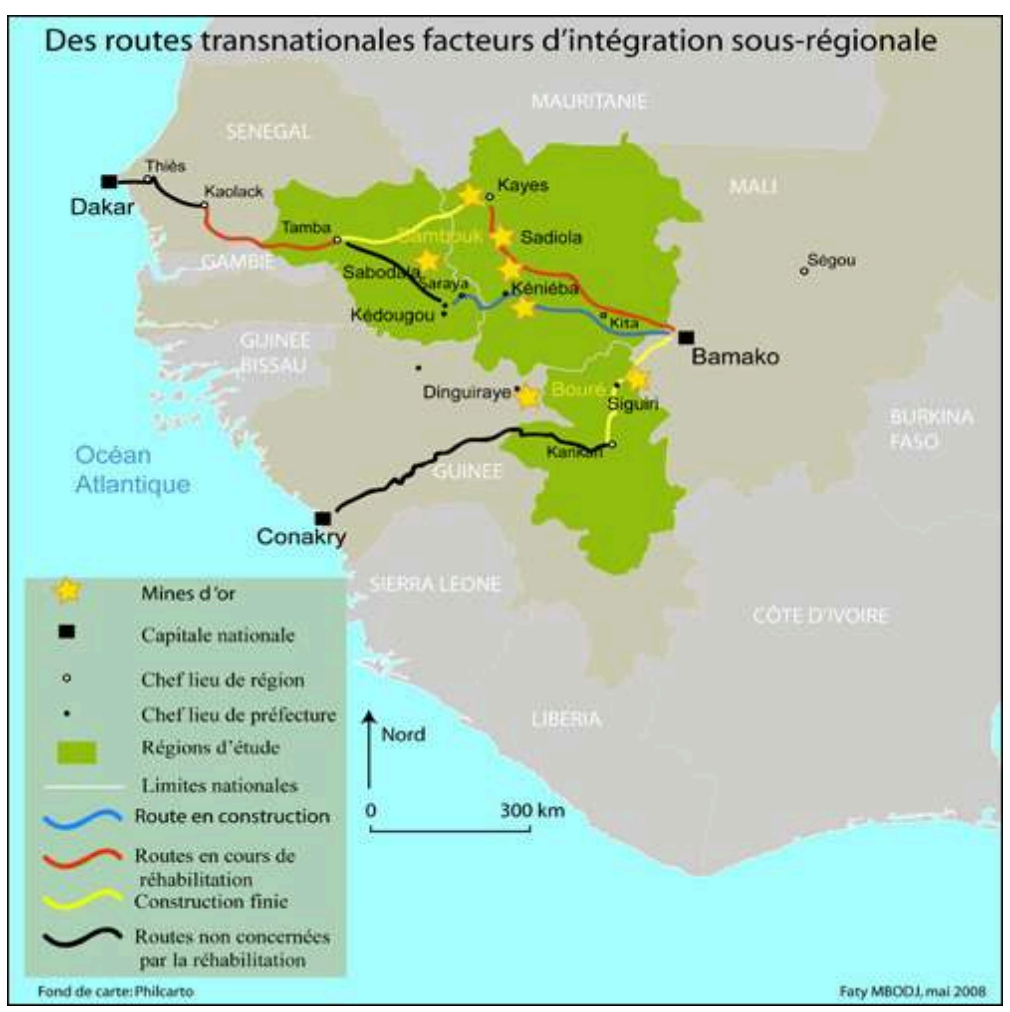

12 En effet, la route Tambacounda-Kayes est goudronnée ; celles de Kayes-Bamako ainsi que Tambacounda-Kaolack sont en voie de réhabilitation. D'autres tronçons dont celui de Kédougou-Bamako, en passant par Saraya (Sénégal), Kéniéba et Kita (Mali), sont également en cours de réalisation. Quant au tronçon Bamako-Kankan, en passant par Siguiri, il est achevé depuis 2004. Il joue un rôle structurant dans les échanges entre la Guinée et le Mali, mais surtout dans le développement socio-économique et le désenclavement de la ville de Siguiri, devenue un important pôle économique régional en haute Guinée.

13 En outre, le contexte du conflit ivoirien a joué en faveur des espaces qui intéressent notre étude. Il a créé les conditions d'une restructuration des flux de marchandises et de personnes en faveur du port de Conakry et surtout de celui de Dakar, par où transitent désormais $70 \%$ des produits européens à destination du Mali ; auparavant, le port d'Abidjan occupait cette position depuis la dislocation de la confédération du Mali en 1960. Une des principales conséquences de cette nouvelle donne est la redynamisation des espaces transfrontaliers situés entre l'est du Sénégal et l'ouest du Mali $^{3}$. Ils deviennent des zones de transit en position favorable à l'expansion de l'économie locale.

14 Parallèlement à ces mutations régionales, les activités extractives vont, elles aussi, constituer d'importants facteurs de changement autant à travers les investissements liés aux fonds qu'elles allouent aux populations riveraines, que par les transformations indirectes (activités annexes) qu'elles occasionnent. 


\section{Transformations liées à l'exploitation industrielle : un nouveau tournant?}

15 Alors que les collectivités locales peinent à profiter de l'autonomie et des ressources financières que la décentralisation est censée leur apporter, les multinationales de l'or se positionnent comme les premiers bailleurs des projets de développement locaux grâce aux fonds qu'elles alimentent régulièrement. Aussi leurs activités génèrent-elles des dynamiques commerciales, démographiques et spatiales nécessaires à l'expansion des économies locales.

\section{Investissements miniers au service du développement communautaire}

Dans chacune des zones étudiées, des fonds de développement communautaire sont alimentés par les compagnies minières. Dans la commune de Sadiola, il s'agit de 5000 dollars mensuels versés par la SEMOS depuis 1997 et Yatéla depuis 2000. En 2004, les mensualités ont augmenté de 3000 dollars, pour atteindre 8000 dollars. A Siguiri, ils correspondent à $0,4 \%$ du chiffre d'affaires annuel de la SAG. A Sabodala, la MDL (Minéral Deposit Limited) déploie 425000 dollars par an.

Ces fonds ont permis la réalisation de plusieurs infrastructures (écoles, forages et puits, postes de santé, banques de céréales, formations pour l'alphabétisation, mosquées etc.). Toutefois, trois problèmes se posent quant à leur gestion et leurs effets sur le développement économique.

Premièrement, ils sont territorialisés, c'est-à-dire qu'ils portent souvent uniquement sur les villages situés dans les périmètres des sociétés; cela entraine une inégalité spatiale et des rivalités. Par exemple, sur les 46 villages que compte la commune de Sadiola, seuls 17 sont concernés par les investissements miniers: 8 villages de la SEMOS ; 7 de Yatéla ; deux autres (Kakadian et Babala) sont identifiés comme villagestests dans le cadre du PADI (Programme d'Appui au Développement Intégré de Sadiola) qui, depuis 2005, gère les fonds destinés à la collectivité.

Deuxièmement, même si à l'échelle locale les investissements paraissent considérables, ils sont faibles comparés aux flux financiers en jeu. Par exemple, sur les 1207 milliards FCFA de chiffre d'affaires de la SEMOS entre 1997 et 2007, la part qui revient aux communautés locales à travers les investissements sociaux n'est que de 9 milliards, soit $0,75 \%$. 
Tableau 1 - Affectation du chiffre d'affaires de la SEMOS (1997-2007)

\begin{tabular}{|l|r|r|}
\hline Dépenses et investissements & 621 milliards & $51,45 \%$ \\
Etat malien & 242 milliards & $20,05 \%$ \\
Anglogold lamgold et IFC & 157 milliards & $13,01 \%$ \\
Fournisseurs & 162 milliards & $13,42 \%$ \\
Salaires locaux & 16 milliards & $1,33 \%$ \\
Investissements sociaux & 9 milliards & $0,75 \%$ \\
Total & 1207 milliards & $100,00 \%$ \\
\hline
\end{tabular}

Source : SEMOS, 2008.4

Troisièmement, ils sont peu porteurs de développement durable en ce sens que les activités génératrices de revenus, capables de favoriser l'autonomie des riverains après la mine, y sont faiblement considérées. Les investissements sont essentiellement centrés sur la résolution de problèmes ponctuels et non sur des perspectives économiques durables.

Certes, dans chacune des zones étudiées, des structures sont mises en place pour un investissement plus pertinent des fonds sociaux versés par les sociétés minières. Il s'agit du PSM (Programme Social Minier) pour Sabodala, de la CGA (Cellule de Gestion Autonome) pour Siguiri et du PADI pour Sadiola. Mais ces structures de contrôle restent confrontées à des difficultés.

2 En effet, le PSM, récemment élaboré (janvier 2008) pour une durée de cinq ans, dispose d'un budget de $3600000000 \mathrm{FCFA}$, soit 5500000 euros $^{5}$. Toutefois, on peut constater quelques contradictions dans sa construction théorique. Ainsi, dans les différents digrammes synthétiques de la répartition des investissements par commune et dans la synthèse générale, le secteur agricole et les activités génératrices de revenus n'apparaissent nullement. Pourtant dans la phase «programmation des investissements ", il était prévu d'appuyer les paysans par l'achat de petits matériels agricoles ou d'intrants, la construction de bassins de rétention, l'aménagement de terres et de mini-barrages, la mise en place de lignes de crédit pour financer les activités génératrices de revenus, etc. Or, apparemment, c'est en réalité essentiellement dans les infrastructures sociales (hydraulique, aménagement de pistes et éducation) que ces fonds seront investis.

Quant à la CGA, à Siguiri, elle est confrontée à des problèmes financiers qui remettent en question son autonomie. Les membres sont payés mais leurs outils de travail (groupe électrogène, ordinateurs, photocopieuses, téléphones) ne fonctionnent plus régulièrement faute de moyens financiers. La SAG et la préfecture se renvoient la responsabilité quant au déploiement des fonds.

Le PADI semble rester la seule structure qui, pour le moment, fait preuve de gestion dans une optique durable en investissant surtout sur le micro-crédit, le maraîchage, l'apiculture, l'agriculture (avec la promotion des espèces améliorées pour accroître les rendements), etc. Mais son avenir est évidemment lié à celui de la SEMOS.

5 Au-delà de cette incidence directe, les activités extractives entrainent d'autres changements grâce aux transformations indirectes induites par les sociétés minières. 


\section{Mutations indirectes occasionnées par l'implantation des multinationales} commerciaux de la haute Guinée. Les flux de marchandises y convergent, entre autres, depuis Conakry, Kankan, la Guinée forestière (Nzérékoré), Bamako (Mali), etc. Aussi, les échanges commerciaux s'intensifient-ils d'une part, entre Siguiri et certains villages de la sous préfecture de Kintinian proches du centre minier et, d'autre part, entre ceux-ci et le reste de la Guinée. Par exemple, les voitures en provenance de Nzérékoré (Guinée forestière) qui s'arrêtaient auparavant à Siguiri arrivent désormais jusque dans les villages cités pour commercialiser l'huile de palme, l'avocat, les bananes plantain, l'igname, le manioc etc. 
Photographies 1 (à gauche) et 2 (à droite) - Marché hebdomadaire de Kintinian

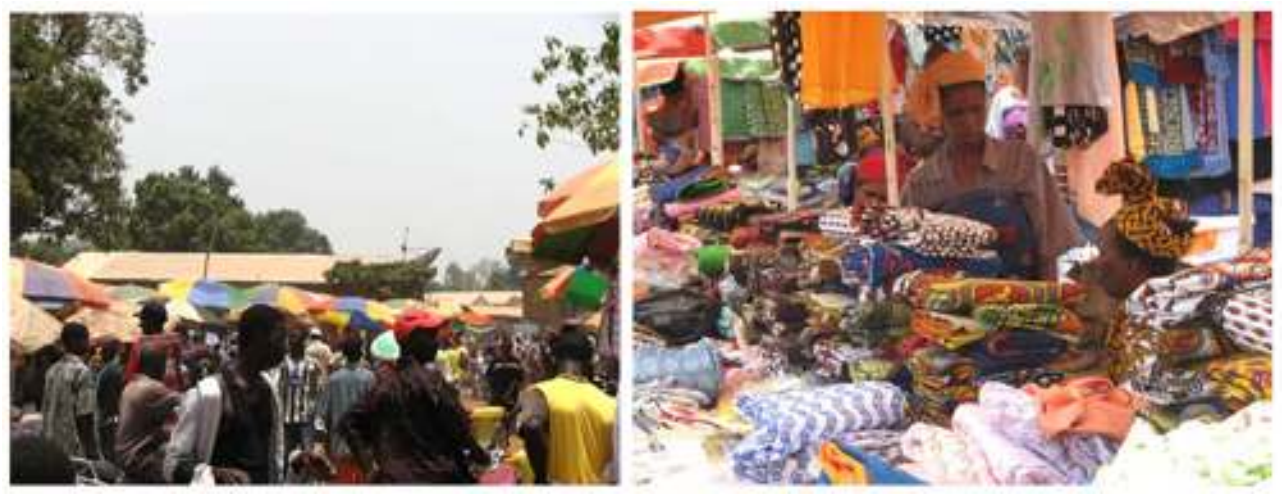

Le marché de Kintinian est ouvert tous les jours. Mais c'est le jeudi qu'il reçoit le maximum de personnes ainsi que diverses marchandises. Sur la photo $n^{\circ} 2$ figurent des vendeuses de pagnes venant de Bamako.

Clichés : Faty B. Mbodj, avril 2008.

Photographies 3 (à gauche) et 4 (à droite) - Camions de commerçantes venant de Guinée forestière
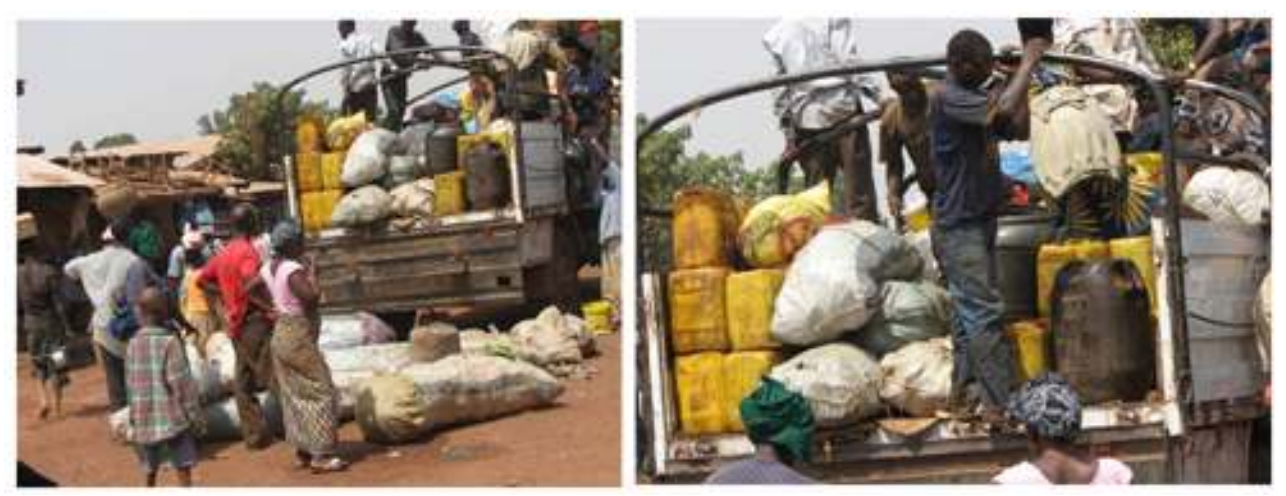

Sur les deux photos ci-dessus des commerçantes, venant de la Guinée forestière, s'apprêtent à charger le reste de leurs marchandises (bananes plantain, huile de palme, avocat etc.) pour d'autres marchés hebdomadaires de la région.

Clichés : Faty B Mbodj, avril 2008.

31 La dynamique spatiale de Siguiri est manifeste dans le domaine immobilier, devenu le principal secteur d'investissement des populations. Les constructions modernes surgissent de toutes parts, stimulées par la facilitation de l'accès au crédit bancaire pour les travailleurs de la SAG. Sous la garantie de cette dernière, la Société Générale de Guinée accorde un crédit à tout employé qui présente un projet fiable. 

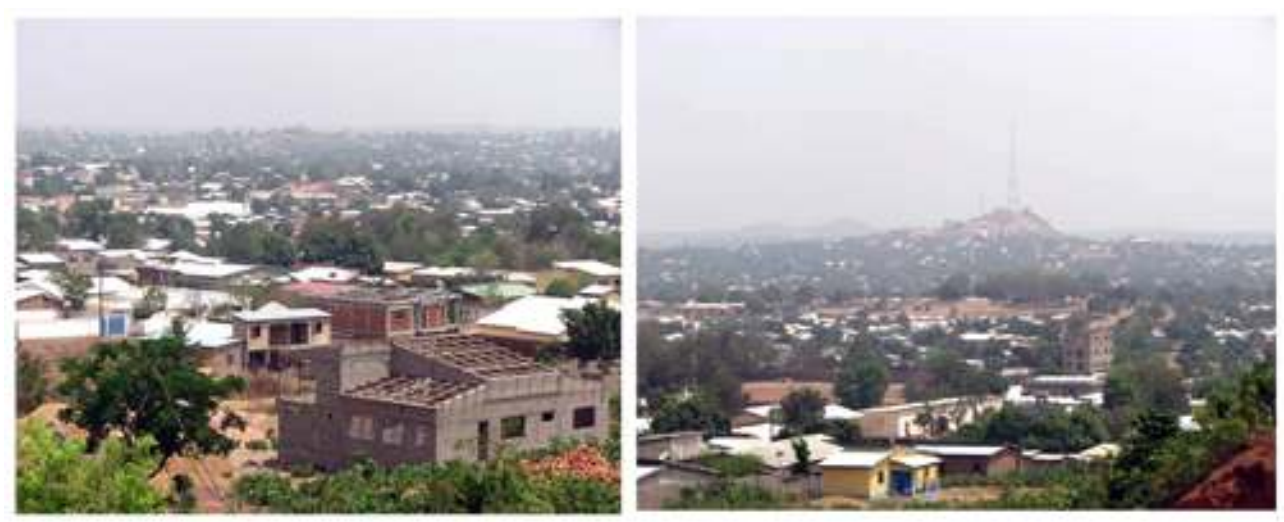

Ces photos, prises à l'entrée de la ville de Siguiri, indiquent l'expansion du secteur immobilier par la multiplication des nouvelles constructions dont des hôtels de luxe. Elles sont surtout le fait des commerçants de l'or, des employés de la SAG ainsi que divers autres types de résidents de la ville.

Clichés : Faty B Mbodj, avril 2008.

Les transformations liées à l'activité minière ne se limitent pas à l'échelle locale. Elles s'étendent aussi au niveau sous-régional où elles jouent un rôle considérable dans l'intensification des échanges entre le Sénégal et le Mali puisque plus de la moitié des mines maliennes se trouvent dans la région de Kayes, le long de la falaise de Tambaoura (voir carte de présentation). En raison de cette proximité géographique, toutes les sociétés minières implantées dans cette zone (Yatéla, Sadiola, Tabakoto, Loulo) s'approvisionnent à Dakar et y font transiter leurs marchandises. Il s'agit de produits alimentaires (à l'exception de la viande), d'hydrocarbures (gasoil) ou encore de produits chimiques nécessaires au processus d'extraction de l'or (cyanure, carbone, metabisulphite, HCL, sulfate de cuivre, ciment, boules en fer servant à écraser le cyanure). Par exemple, pour le traitement du minerai de Yatéla, 100 tonnes de ciment sont importées quotidiennement du Sénégal. Cela contribuerait à la création d'une troisième cimenterie au Sénégal.

Certes, les contributions financières des multinationales font l'objet d'une gestion souffrant de défaillances, attribuées à la faible capacité de positionnement des collectivités face aux puissances industrielles. Pourtant, ces transformations indirectes révèlent que les zones minières du Bouré et du Bambouk sont en passe de pérenniser les dynamiques économiques actuellement observées. Quant aux interactions entre activités extractives et agriculture, elles apparaissent ambivalentes.

\section{Activités extractives et économies agricoles}

L'entrée en vigueur de la production des multinationales de l'or correspond à une phase de crise des cultures de rente (coton et arachide) qui structuraient les économies nationales et locales des pays et régions étudiées. Cette production constitue un moteur de bouleversement des relations agriculture-exploitation minière (artisanale) parce qu'elle entraine des pertes de terres de culture, de la pollution ou encore l'abandon de l'agriculture par les populations riveraines espérant trouver un emploi minier etc. Mais elle offre toutefois des perspectives de stimulation du secteur vivrier marchand afin de satisfaire la demande d'une population croissante. 


\section{Des économies agricoles en réelle difficulté}

Autant au Sénégal qu'au Mali et en Guinée, les économies agricoles des zones étudiées sont en grande difficulté. Les cultures de rente (l'arachide pour Sadiola, le coton à Sabodala et Siguiri) constituaient les principales sources de revenus pour les paysans. Or, depuis plusieurs années, elles sont soumises à des contraintes externes et internes (baisse des cours mondiaux, faible niveau d'encadrement, caution solidaire ${ }^{8}$ etc.) qui accentuent leurs difficultés au point de causer parfois leur disparition. C'est le cas à Sadiola où, depuis les années 1980, l'arachide n'est plus commercialisée et où les structures d'encadrement pour les paysans ont disparu. A ces difficultés existantes s'ajoutent celles liées aux activités des multinationales qui exercent une concurrence directe et indirecte sur les économies agricoles.

\section{Concurrence entre mines et agriculture}

Dans l'histoire économique ancienne des régions étudiées, il n'y a jamais eu de concurrence entre activités minières et agriculture. Les deux ont toujours fonctionné en binôme complémentaire: l'orpaillage n'était pratiqué qu'en saison sèche, l'hivernage était réservé à l'agriculture. Pourtant, dans la CR (communauté rurale) de Khossanto, une étude montre que la période 2004-2006 a été difficile pour les cultures cotonnières et vivrières (Dia, 2006). En effet, l'implantation des sociétés minières, dans un contexte de crise du coton, provoque le bouleversement des calendriers qui structuraient jusque là les activités économiques rurales, et instaure un cercle vicieux. Par exemple, un ancien cotonculteur qui décroche un contrat de 6 mois à la mine est sûr de gagner plus que s'il cultivait du coton avec un salaire journalier de 2500 FCFA. Un mois de travail représente 70000 FCFA (un peu plus de 100 euros). Il se retrouve ainsi avec 420000 FCFA soit pratiquement le triple des revenus d'une production d'un hectare de coton de premier choix. De plus, il a la certitude d'obtenir son salaire à la fin du mois, alors que les activités agricoles sont soumises à de nombreux aléas (climat, attaques des parasites, caution solidaire etc.).

Ainsi, dans le Bélédougou (principauté fondée après la dislocation de l'empire du Mali, correspondant aujourd'hui à la communauté rurale de Khossanto), un fort taux d'abandon de la culture cotonnière a été constaté. Dans certains villages proches du centre minier, il dépasse les $50 \%$ et quelques villages ont tout simplement abandonné le coton. 
Carte 4 - Abandon de la culture du coton dans les villages étudiés

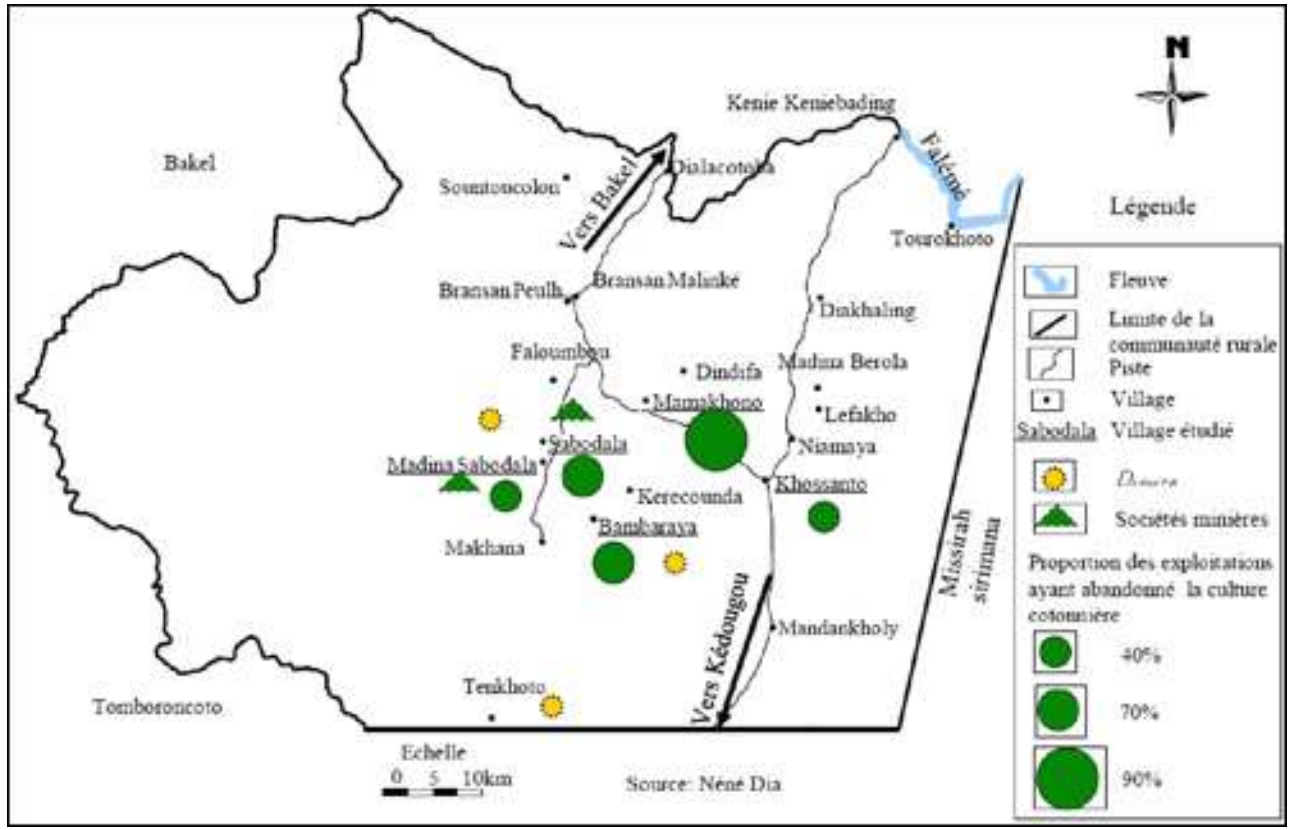

Auteur : Néné DIA, 2006

Pour leur part, les superficies des cultures vivrières ont également subi une forte baisse. C'est d'ailleurs dans ce lien secteur vivrier-coton que réside la pertinence du système coton en Afrique de l'Ouest : les intrants fournis à destination du coton, par la SODEFITEXE, servent aussi pour augmenter les rendements des céréales.

Diagramme 1 - Réduction des superficies destinées aux cultures vivrières dans les 5 villages étudiés

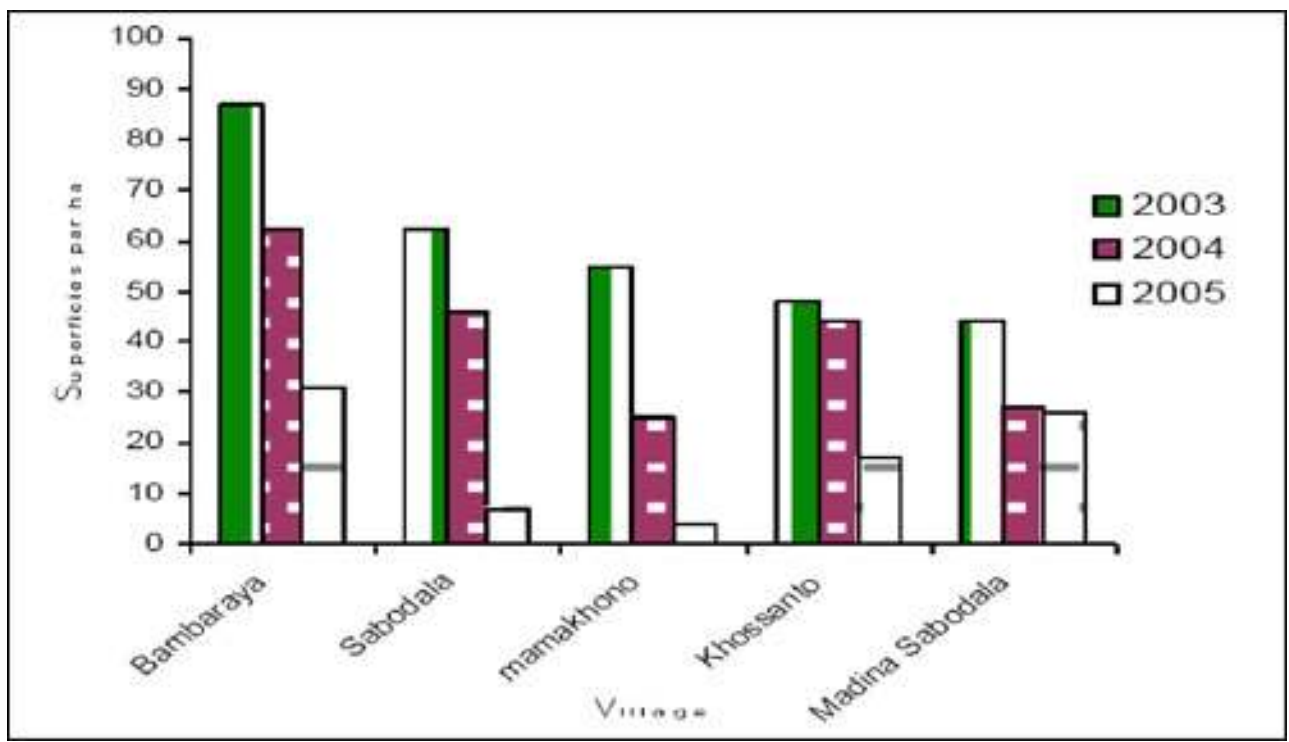

Auteur : Néné DIA, 2006.

Une telle situation entraine un risque de déficit alimentaire pour les paysans de ces villages. Comme la production locale diminue, les prix des ressources vivrières augmentent. L'argent gagné dans les mines et les sites d'orpaillage sert alors 
essentiellement à acheter du riz. Cela se traduit par une accentuation de la modification (déjà entamée sous l'effet des revenus de la culture du coton) des pratiques alimentaires, traditionnellement fondées sur le mil.

Dans la CRD (communauté rurale de développement) de Kintinian, les activités extractives de la SAG perturbent considérablement l'agriculture, à travers notamment la baisse des superficies cultivables et les nombreux cas d'expropriation qu'elles occasionnent. En effet, c'est dans le cadre légal des possibilités offertes aux sociétés minières par le code minier que les actes d'expropriation de champs de cultures se font. La valeur terre n'est pas compensée, mais les cultures qui y étaient effectuées le sont ; cela signifie que les terres en jachère sont exclues du processus de dédommagement. Le service de l'agriculture de la préfecture est chargé de déterminer le rendement à l'hectare pour le type de culture effectué par le paysan, ainsi que son prix au kg. Sur la base de ces deux indices, les bénéfices probables sont estimés et les compensations faites.

Par ailleurs, la superficie du barrage à boue mis en place par la SAG est d'environ 400 hectares. Aussi entre 2005 et 2007, 352 champs, soit environ 123 hectares, ont été expropriées. Certes, les paysans ont bénéficié de dédommagements. Selon la SAG, le coût de compensation pour ces trois années équivaudrait à environ 1,15 millions de dollars. Toutefois, l'impact négatif sur l'agriculture s'avère évident sachant que ces montants sont essentiellement réinvestis dans l'immobilier (dans la ville de Siguiri) et rarement dans l'agriculture'.

43 A Sadiola aussi, les cultures vivrières sont, à certains endroits, affectées par les activités de la SEMOS. Nous ne disposons pas de chiffres sur le nombre de champs expropriés, mais la superficie couverte par le barrage à boue est de 320 hectares. Cet espace, qui constituait la zone de culture de quatre villages (Sadiola, Farabacouta, Tabakoto et Sekokoto), est désormais englouti dans le périmètre de la SEMOS et semble être à jamais impropre à l'agriculture et au pâturage, même après la fin de l'exploitation. La pollution au cyanure y est en effet d'autant plus forte que le sol n'est pas tapissé par une couche imperméable. Les populations de ces villages se plaignent, d'une part, de l'éloignement de leurs nouveaux champs, et d'autre part, de la faible fertilité des terres. Il arrive aussi que les sociétés n'exproprient pas les paysans mais affectent leur production de manière indirecte. Les activités de prospection nécessitent en effet le déblayage des terrains choisis. Or la fertilité du sol dépend de la couverture de surface ; ainsi, même une fois les travaux finis, les paysans doivent patienter quelques temps avant de retrouver la fertilité de leurs terres.

Ces processus contribuent à une réduction des temps de jachère et probablement à celle des rendements, notamment dans des systèmes de production où cette stratégie est la principale forme de fertilisation des terres. Si de tels exemples traduisent une vulnérabilité de l'agriculture face aux activités extractives, celles-ci constituent parfois aussi un stimulant pour le vivrier marchand.

\section{Le vivrier marchand à l'heure de l'exploitation industrielle (Siguiri et Sadiola)}

La dynamique que connait aujourd'hui l'économie du vivrier marchand à Siguiri et Sadiola n'est guère induite par la part de consommation des sociétés minières en fruits 
et légumes, issus du milieu local, qui reste très faible. Celles-ci préfèrent s'approvisionner dans les capitales nationales (Dakar, Bamako, Conakry). En fait, leur rôle dans le dynamisme de ce type d'économie réside dans l'accroissement de la population et du pouvoir d'achat qu'elles provoquent.

47 A Siguiri, autant l'exploitation industrielle affecte les superficies agricoles, autant elle est devenue un facteur stimulant pour l'arboriculture. Car les paysans sont en permanence informés de l'évolution géographique des activités de la SAG. En fonction de cela, ils plantent des centaines de pieds d'anacardiers et de manguiers; c'est une stratégie pour matérialiser la mise en valeur de leur capital foncier pour bénéficier de compensations en cas d'opérations de déguerpissement. L'une des conséquences de cette mesure préventive est l'intensification de la culture de l'anacardier dans tout le Bouré. Les noix de cajou qui en sont issues sont commercialisées au-delà de la souspréfecture.

48 En dehors de l'arboriculture, les activités extractives ont stimulé le maraîchage. Il s'est développé dans les environs immédiats de Siguiri, mais aussi dans d'autres villages disposant d'eau, parfois situés à des dizaines de kilomètres sur la route transnationale. Par exemple, Tougui Oulen, village situé à 10 kilomètres sur la route Siguiri-Kankan, a une longue tradition de maraîchage. Mais jusque là cette activité était réservée aux femmes. Aujourd'hui, les hommes aussi s'y sont mis étant donné l'intérêt qu'elle représente.

49 A Sadiola, ce sont surtout les allochtones qui ont saisi l'opportunité de la pratique du maraîchage, en initiant un processus d'appropriation de terres non utilisées par les autochtones. Les « étrangers » qui pratiquent l'activité n'ont pas tous le même statut et peuvent être classés en trois catégories. La première est constituée par ceux qui sont venus chercher du travail à la mine, en vain. Ils se sont alors engagés comme ouvriers agricoles. La deuxième catégorie englobe ceux qui n'ont pas décroché d'emploi mais ont réussi à se procurer une petite parcelle pour eux-mêmes. Quant à la troisième catégorie, elle rassemble ceux que l'on peut qualifier de travailleurs-entrepreneurs. Il s'agit des employés de la mine qui, tout en conservant leurs postes à la SEMOS, disposent d'une parcelle et emploient de la main-d'œuvre pour y travailler ; eux-mêmes assurant le rôle de superviseurs.

Aujourd'hui, le centre de Sadiola est donc autosuffisant en produits maraîchers et ravitaille les villages environnants. Une partie de sa production est acheminée sur le marché de Kayes dont il était autrefois dépendant.

51 Cette expansion du vivrier marchand est un des facteurs qui témoignent des dynamiques de développement liées à l'implantation des multinationales dans le Bouré et le Bambouk.

\section{Conclusion}

52 Certes, les activités extractives dans les territoires du Bouré et du Bambouk constituent des facteurs favorables au développement et à l'intégration de ces espaces en marge, grâce aux transformations directes ou indirectes qu'elles entrainent, et notamment à la stimulation de l'économie du vivrier marchand. Mais les retombées à l'échelle locale ne sont pas à la hauteur de la valeur économique des ressources aurifères extraites, notamment dans le contexte actuel. L'insuffisance de ces retombées hypothèque 
l'avenir économique des régions aurifères étudiées, d'autant que les ressources en question ne sont pas renouvelables. Les économies agricoles sont en crise et l'orpaillage est devenu le parent pauvre de l'exploitation industrielle.

Dans certains cas comme le Sénégal (qui vient de débuter l'exploitation de son potentiel aurifère), le cours des évènements peut prendre une autre tournure si des efforts sont déployés en faveur d'un renforcement des capacités de négociations des riverains, d'une augmentation des retombées locales de l'exploitation, mais aussi de l'orientation de celles-ci vers des modes d'investissement plus durables.

Dans le contexte actuel plus que jamais, les gouvernants des Etats miniers doivent profiter du caractère stratégique de leurs ressources. En effet, malgré l'intensité de la crise économique générale en cours, l'or est épargné et représente une valeur-refuge qui justifie l'augmentation continue de son prix.

\section{BIBLIOGRAPHIE}

CGA, 2008. Rapport d'activité de la Cellule de gestion autonome de Siguiri de 2007. Siguiri, 33 p.

Dia N, 2006. Activité minière et culture cotonnière : l'exemple de la mine d'or de Sabodala au Sudest du Sénégal. Mémoire de maitrise. UGB Saint-Louis, 98 p.

Girard J, 1992. L'Or du Bambouk : du royaume de Gabou à la Casamance une dynamique de civilisation ouest-africaine, Genève, Georg, 347 p.

Mbodj, M, 1992. « La crise trentenaire de l'économie arachidière », Diop M C (dir), Le Sénégal trajectoire d'un Etat. Dakar, Codesria, p. 93-135.

Ninot 0, 2003.«vie de relations, organisation de l'espace et développement en Afrique de l'Ouest : la région de Tambacounda au Sénégal ». Thèse de géographie. IRD, Université de Rouen. 460 pages.

Programme des Nations Unies pour le développement/PNUD, 2001. « Rapport mondial sur le développement humain. Mettre les nouvelles technologies au service du développement humain », Paris, Economia, 264 pages.

Service régional chargé de la planification et agence régionale de développement, 2008. Programme social minier. Tambacounda, 149 pages.

Tropica Environmental Consultants, 2006. Etude d Impact Environnemental et Social du projet d'exploitation d or, d argent et de substances connexes de Sabodala (rapport provisoire), volume 2-PGES et Annexes, Dakar, $72 \mathrm{p}$.

UEMOA, BCAO, 2006. Programme économique régional (PER) 2006-2010. Volum IV, fiches de projets. Juillet 2006, 289 p. 


\section{NOTES}

1. Le Bambouk se trouve à cheval entre le Mali et le Sénégal. Quant au Bouré, il est localisé dans la préfecture de Siguiri et précisément dans la communauté rurale de Kintinian. Ces provinces ont une identité aurifère assez forte et leur or a joué un rôle déterminant dans l'histoire politique des grandes structures étatiques pré-coloniales, notamment les empires du Ghana et du Mali.

2. La mine se trouve, certes, à Kintinian mais autant les chercheurs, les politiques que les autres utilisent généralement le nom de Siguiri pour la désigner.

3. Entretien avec Mme Traoré, directrice de l'entrepôt du Mali au Sénégal, avril 2008.

4. Anglogold (sud africaine) et Iamgold (canadienne) sont chacune actionnaire jusqu'à hauteur $38 \%$. L'IFC qui représente la Banque mondiale détient $6 \%$ de part d'action tandis que les $18 \%$ restant reviennent à l'Etat malien.

5. Ce montant inclut la contribution d'autres sociétés telles que la canadienne OROMIN et le groupe MITAL STEEL qui devait débuter en 2009 l'exploitation d'un important gisement de fer dans la région.

6. SEMOS : société d'exploitation des mines d'or de Sadiola.

7. Selon le recensement général de 1976, la taille de la population de l'ensemble de la commune (46 villages) était de 12400 habitants. Pendant le recensement général de 1998, elle était de 19400 personnes. Quant aux données de 2007, elles sont fournies par la sous-préfecture qui estime la population de la commune à 21000 personnes.

8. Le système de caution solidaire constitue un véritable handicap pour l'économie cotonnière. Car lorsqu'un membre du groupement de producteurs n'arrive pas à rembourser ses dettes, les autres doivent le faire à sa place ; c'est la condition pour que le groupement puisse bénéficier d'un crédit pour la prochaine campagne agricole. Par ailleurs, même si tous les cotonculteurs respectent leur engagement, ce n'est qu'à la fin de la campagne qu'ils peuvent obtenir leur argent, donc toujours tardivement; ce qui n'est pas le cas avec les emplois miniers.

9. Entretien avec M Camara, le président de la CRD de Kintinian ; avril 2008.

\section{RÉSUMÉS}

La conjoncture mondiale et les réformes minières entreprises par des pays comme la Guinée (1995), le Mali (1999) et le Sénégal (2003) ont créé les conditions favorables à l'investissement de multinationales de l'or dans un espace transfrontalier situé entre l'est du Sénégal, l'ouest du Mali et le nord-est de la Guinée. A travers les activités annexes et les investissements directs occasionnés, ces multinationales apparaissent comme un facteur d'intégration nationale et sous régionale de ces zones aurifères jusqu'ici en marge. Toutefois, l'insuffisance de leurs contributions financières, la gestion peu efficace de celles-ci, ainsi que la concurrence directe et indirecte que subissent les économies agricoles soulèvent des interrogations sur l'avenir économique des régions étudiées face aux activités extractives.

The mining reforms undertaken by countries like Guinea (1995), Mali (1999) and Senegal (2003) have created favorable conditions for the investment of gold multinationals in a transborder area located between the east of Senegal, the west of Mali and the north-east of Guinea. Through the side activities and direct investments brought about, these firms appear as an opportunity to integrate these marginalized zones within the national and regional economies. However, their 
financial contributions are insufficient and they provoke direct and indirect competition on local agricultural economies, raising questions on the role of extractive activities for the economic future of the areas studied.

INDEX

Keywords : enclave, extractive activity, gold resource, rural economy, transformation

Mots-clés : activité extractive, économie rurale, enclave, ressource aurifère, transformation

\section{AUTEUR}

\section{FATY B. MBODJ}

Faty B. Mbodj est doctorante en géographie à l'Université de Paris1 Sorbonne et à l'Université Gaston Berger de Saint-Louis du Sénégal. Elle est membre de l'UMR CNRS PRODIG et associée au CIRAD. 